\title{
A note on prime zeta function and Riemann zeta function. Corrigendum
}

\section{Mladen Vassilev-Missana}

\author{
V. Hugo 5 Str, 1124, Sofia, Bulgaria \\ E-mail: statiamatheabv.bg
}

Received: 21 April 2021

Revised: 3 June 2021

Accepted: 7 June 2021

Abstract: In [1] the author proposed two new results concerning the prime zeta function and the Riemann zeta function but they turn out to be wrong. In the present paper we provide their correct form.

Keywords: Prime zeta function, Riemann zeta function, Prime numbers.

2020 Mathematics Subject Classifications: 11A25, $11 \mathrm{M} 06$.

\section{Introduction}

Let $\zeta(s)$ denote the Riemann zeta, i.e.

$$
\zeta(s)=\sum_{k=1}^{\infty} \frac{1}{k^{s}},
$$

where $s \in \mathbb{C}(\mathbb{C}$ is the set of complex numbers) and $\Re(s)>1$. Let $P(s)$ be the prime zeta function:

$$
P(s)=\sum_{p} \frac{1}{p^{s}},
$$

where $s \in \mathbb{C}$ and $\Re(s)>1$, and $p \in \mathbb{P}$ ( $\mathbb{P}$ is the set of all primes).

In [1] the following two results were claimed as true:

Theorem 1 ([1]). For integer $s>1$, the following recurrent relation

$$
(1-P(s))^{2}=\frac{2}{\zeta(s)}-1+P(2 s) .
$$

holds.

And as a Corollary:

$$
P(s)=1-\sqrt{\frac{2}{\zeta(s)}-1+P(2 s)} .
$$


Theorem 2 ([1]). Prime zeta function $P(s)$ for every fixed integer $s>1$, could be expressed with the help of the values of Riemann zeta function $\zeta\left(2^{k} . s\right), k=1,2,3, \ldots$, by the formula

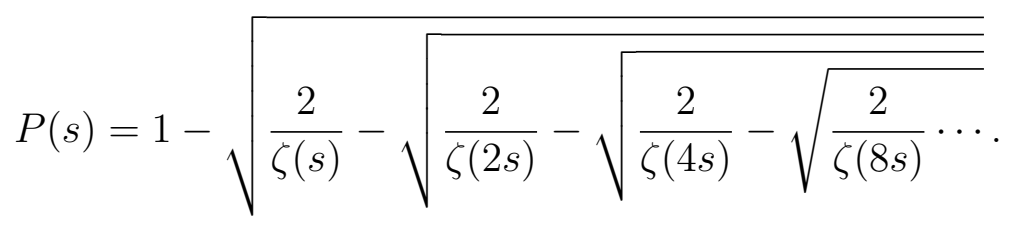

Recently, Richard P. Brent disproved the stated above Theorems (and Corollary), [2].

\section{Correct form of the theorems}

Below, we introduce some denotations and give the correct form of our Theorems.

Let

$$
P_{m}(s) \stackrel{\text { def }}{=} \sum \frac{1}{\left(p_{i_{1}} p_{i_{2}} \cdots p_{i_{m}}\right)^{s}}
$$

where $m \geq 2$ and $p_{i_{1}}<p_{i_{2}}<\cdots<p_{i_{m}}$ run over $\mathbb{P}$.

$$
\varepsilon(s) \stackrel{\text { def }}{=} \sum_{m=3}^{\infty}(-1)^{m-1} P_{m}(s) .
$$

In (2) and (3) $s \in \mathbb{C}$ and $\Re(s)>1$. Then we provide the correct form of [1, Theorem 1] as follows

Theorem 3 (Correct form of Theorem 1). For $s \in \mathbb{C}$ and $\Re(s)>1$, the following recurrent relation

$$
(1-P(s))^{2}=\frac{2}{\zeta(s)}+2 \varepsilon(s)-1+P(2 s)
$$

holds.

For real $s>1$ from (4) we obtain (since $0<P(s)<1$ ):

\section{Corollary 1.}

$$
P(s)=1-\sqrt{\frac{2}{\zeta(s)}+2 \varepsilon(s)-1+P(2 s)} .
$$

Theorem 4 (Correct form of Theorem 2). The representation

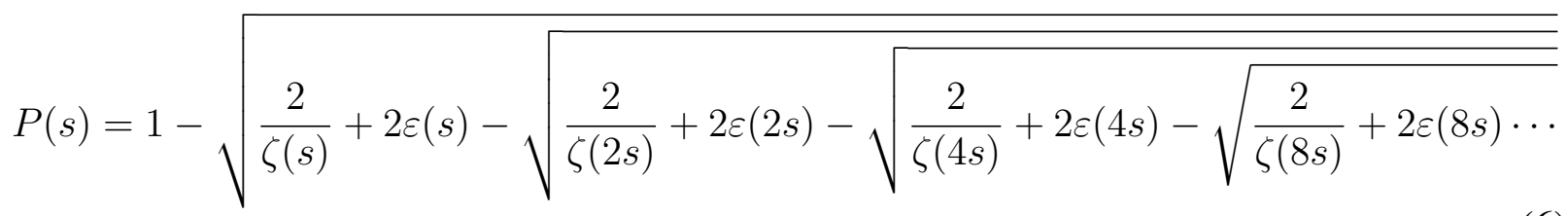

holds for real $s>1$.

Since (6) follows directly from (5), it suffices to prove Theorem 3.

Proof of Theorem 3. We obviously have

$$
\frac{1}{\zeta(s)}=\sum_{m=1}^{\infty} \frac{\mu(m)}{m^{s}}
$$

where $\mu$ is the Möbius function. 
Hence,

$$
\frac{1}{\zeta(s)}=1-P(s)+P_{2}(s)-\varepsilon(s)
$$

(see (2) and (3)).

Now we use that

$$
P_{2}(s)=\frac{(P(s))^{2}-P(2 s)}{2} .
$$

The last equality and (7) yield (4) and Theorem 3 is proved.

\section{Final comments}

1) Using the well known representation:

$$
P(s)=\sum_{k=1}^{\infty} \frac{\mu(k)}{k} \log (\zeta(k s))
$$

(see e.g. [3-5]), it is clear that we may express $\varepsilon(s)$ with the help of $\zeta(s)$ because of (4) and the fact that

$$
P(2 s)=\sum_{k=1}^{\infty} \frac{\mu(k)}{k} \log (\zeta(2 k s)) .
$$

So in the right hand-side of (6) only values of Riemann's zeta function will appear.

2) Let us note, that formula (1) is an approximation of the prime zeta function $P(s)$ when $s>1$ is a real number, since

$$
\lim _{s \rightarrow+\infty} \varepsilon(s)=0
$$

\section{Acknowledgements}

The author is thankful to Richard P. Brent, who observed that [1, Theorems 1 and 2] are wrong, and thus helped the author to find the correct forms of these theorems.

\section{References}

[1] Vassilev-Missana, M. (2016). A note on prime zeta function and Riemann zeta function. Notes on Number Theory and Discrete Mathematics, 22(4), 12-15.

[2] Brent, R. P. (2021). On two theorems of Vassilev-Missana, Notes on Number Theory and Discrete Mathematics, 27(2), 49-50.

[3] Glaisher, J. W. L. (1891). On the sums of inverse powers of the prime numbers. The Quarterly Journal of Mathematics, 25, 347-362.

[4] Fröberg, C.-E. (1968). On the prime zeta function. BIT Numerical Mathematics, 8, 187-202.

[5] Cohen, H. (2000). Advanced Topics in Computational Number Theory. New York: Springer-Verlag. 